\title{
ESTRATEGIAS METACOGNITIVAS DE COMPRENSIÓN DE LECTURA EN EL AULA DE ESPAÑOL COMO SEGUNDA LENGUA O LENGUA EXTRANJERA
}

Ana Ligia López Jurado

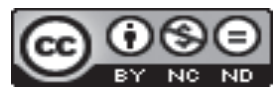

Esta obra está bajo una licencia Creative Commons 



\title{
ESTRATEGIAS METACOGNITIVAS DE COMPRENSIÓN DE LECTURA EN EL AULA DE ESPAÑOL COMO SEGUNDA LENGUA O LENGUA EXTRANJERA
}

\author{
METACOGNITIVE STRATEGIES OF READING COMPREHENSION \\ IN THE SPANISH AS A SECOND OR FOREIGN LANGUAGE \\ CLASSROOM
}

\begin{abstract}
RESUMEN
En este artículo se expone qué son y cómo se pueden implementar las estrategias metacognitivas de comprensión de lectura en una clase de español como segunda lengua (L2) o como lengua extranjera (ELE). Se toman en cuenta los tres componentes de la metacognición: el conocimiento declarativo, procedimental y condicional aplicados en el aula como procesos metacognitivos: planificación, monitoreo y control y evaluación. Estas estrategias promueven la autonomía del estudiante al monitorear, controlar y evaluar su propio proceso de aprendizaje (Hall, 1991; Pintrich, 2002; Jiménez-Rodríguez, 2004; Muhammand Al-Khateeb, 2011; Alhaqbani \& Riazi, 2012). Además, estas estrategias aplicadas a las segundas lenguas han permitido que los estudiantes tengan un mejor rendimiento en cuanto a la comprensión lectora (Brantmeir, 2003; Yang, 2002; Farrell, 2001). Palabras clave: metacognición, estrategias, comprensión de lectura, segundas lenguas, lenguas extranjeras.
\end{abstract}

Ana Ligia López Jurado

\begin{abstract}
In this paper, metacognitive strategies are not only explained but it is shown how those strategies can be implemented in a Spanish as a Second Language (L2) (or Foreing Language, ELE) classroom too. Three main metacgonitive components are taken into account: declarative, procedimental and conditional knowledges that can be applied in the classroom as metacognitive processes: planification, monitoring and control, and evaluation. Such strategies promote the learners's autonomy by allowing students to monitor, control and evaluate their own learning process (Hall, 1991; Pintrich, 2002; Jiménez Rodríguez, 2004; Muhammand Al-Khateeb, 2011; Alhaqbani \& Riazi, 2012). Furthermore, when those strategies have been applied in foreign language classroom students has obtained better results at reading comprehension tasks (Brantmeir, 2003; Yang, 2002; Farrell, 2001).
\end{abstract}

Key words: metacognition, strategies, reading comprehension, second languages, foreign languages.

\section{Introducción}

Actualmente, se sabe que leer, sobre todo de manera comprensiva, es una actividad cognitiva compleja e intencionada. La lectura depende tanto en la persona que lee como en el texto que va a ser leído (Cuetos-Vega, 2000; Jiménez-Rodríguez, 2004; Hall, 1991). “La lectura

Bach. Ana Ligia López Jurado. Centro Educativo Saint Michael School. Coordinadora del Departamento de Inglés. Costa Rica.

Correo electrónico: lunajurado@gmail.com

Recepción: 15- 05- 2015

Aceptación: 31- 08- 2015 
supone el procesamiento de información de una clase de símbolos que constituyen expresiones en el intercambio comunicativo que tiene lugar a través del lenguaje" (Sainz, 1991, p. 641).

La idea de que la decodificación es la base de la comprensión lectora y de cualquier proceso de lectura no se sostiene pues se ha demostrado que no se necesita decodificar signo por signo para entender una palabra: "[...] la lectura de las palabras comunes pueden ser identificadas en forma holística mientras que las palabras desconocidas o raras se identifican analizándolas en sus elementos mínimos.” (Navarro, 1991). Lo mismo opina Hall (1991): “[...] el contexto semántico facilita la identificación de las palabras. El conocer en buena medida la relación entre las palabras ayuda a la decodificación." (p. 33).

De esta forma, el proceso de lectura y los procesos de comprensión no radican exclusivamente en la decodificación de símbolos: “[...] la lectura es un proceso interactivo que no avanza en secuencia estricta desde las unidades perceptivas básicas hasta la interpretación global de un texto. Por el contrario, el lector experto deduce, simultáneamente, información de varios niveles, integrando al mismo tiempo información grafofonemática, morfémica, semántica, sintáctica, pragmática, esquemática e interpretativa.” (Hall, 1991, p. 36). La misma opinión es compartida por Navarro (1991): "El carácter interactivo de la lectura también se evidencia en el rol desempeñado por factores como el contexto, las expectativas y la base de conocimiento que aporta el lector" (p. 55). En otras palabras:

\footnotetext{
Leer es el proceso de entender el lenguaje escrito. Empieza con el fluir de patrones en la retina y termina (si hay éxito) con una idea definitiva del mensaje que el autor había planeado. De ahí que leer es al mismo tiempo un proceso "de percepción" y "cognitivo". [...] Más allá de eso, un lector habilidoso debe ser capaz de usar información sensorial, sintáctica, semántica y pragmática para cumplir con las tareas. (Rumelhart, 2013, p. 719)
}

Uno de los problemas de la definición tradicional de comprensión de lectura, en la cual se define comprensión como el entendimiento total y completo del significado de un texto (Yang, 2002, p. 18), radica en que se excluye la posibilidad de identificar diferentes niveles de comprensión: comprensión literal, procesos de calificación y síntesis, comprensión inferencias y lectura crítica (Graesser et ál, 2006). De esta manera, la comprensión debería definirse como el entendimiento de las proposiciones o unidades básicas de significado en un texto: "Esto es, una persona tal vez solo se enfoque en comprensión léxica (palabras), mientras otra se involucre en comprensión sintáctica (oraciones), siendo este último nivel más alto que el anterior" (Yang, 2002, p. 19).

Algunas veces se presentan fallos en la comprensión lectora, cuyas causas son múltiples: ausencia de un esquema mental apropiado que facilite reconocer la estructura y contenido del texto, rompimiento de principios pragmáticos: como el conocimiento mutuamente compartido tanto por el lector como por el autor del texto; carencia de pistas textuales para indicar nueva información o información importante; que lo dicho en el texto se contradiga (Graesser et ál, 2006; Puente, 1991).

Ahora bien, en contextos educativos la comprensión de lectura es fundamental: los fallos tienen serias implicaciones. De ahí que sea necesario entender no solo qué caracteriza a los buenos lectores y a los no tan exitosos, sino que todo esto ha generado el desarrollo de investigaciones que dan pistas sobre cómo se pueden mejorar las habilidades de comprensión de lectura en contextos académicos. Aunque en este artículo no se discuten en profundidad las diferencias entre buenos y malos lectores, sí se sabe que los lectores deficientes no cuentan con tanto conocimiento previo sobre el tema del texto, tienen dificultad para relacionar los 
elementos constituyentes, tienen problemas para elaborar inferencias, no están seguros de qué hacer antes de abordarlo ni son capaces de hacer buenas hipótesis o predicciones de lo que se encontrarán a continuación. Además, tiene poco o nulo conocimiento de estrategias metacognitivas (Puente, 1991, p. 105).

Antes de abordar las estrategias metacognitivas, es importante entender qué es metacognición. Este término empezó a tomar forma desde la publicación de Flavell (1979) el cual fue definido como el conocimiento de uno mismo sobre los propios procesos y productos cognitivos, el conocimiento y las experiencias metacognitivas (Flavell, 1979). No obstante, la definición no se quedó ahí: Antonijeck \& Chadwick (1981) consideraron que la metacognición como el grado de conciencia sobre las actividades mentales propias, es decir, sobre el pensamiento y el lenguaje.

En la actualidad, el concepto de metacognición refiere a los pensamientos propios y sobre la propia cognición (Dunlosky y Metcalfe, 2009; Flavell, 1979); implica, como requisito básico, que los individuos reflexionen sobre su propio conocimiento (Hall, 1991; Jiménez-Rodríguez, 2004) para dar lugar a la regulación de esos procesos de pensamiento (El-Hindi, 1997). De esta manera, la base de la metacognición es saber lo que uno sabe: ¿cómo sé que he estudiado lo suficiente para aprender el contenido de esa unidad?, ¿cuáles estrategias puedo usar para recordar esto y aquello? En resumen, la metacognición "depende de la propia habilidad para evaluar o monitorizar los procesos cognitivos propios, como el pensamiento y la memoria" (Jiménez-Rodríguez, 2004, p. 44).

En todo caso, la metacognición no debe ser confundida con cognición. La cognición es el funcionamiento intelectual de la mente humana (Jiménez-Rodríguez, 2004); tiene que ver con ser capaz de recordar, comprender, focalizar la atención y procesar información, extraer información del medio, integrar información, almacenar en la memoria, evaluar la coherencia lógica de los procesos y productos mentales. La metacognición, por su parte, tiene que ver con una serie de estrategias que vigilan o supervisan los procesos realizados por la cognición.

Siguiendo ese orden de ideas, es necesario que el individuo conozca sus propios procesos cognitivos, con el fin de poder programar conscientemente estrategias para el aprendizaje y la memoria, la resolución de problemas y tomar decisiones: es decir, la persona debe ser capaz de autorregular sus procesos mentales. Además, debe ser capaz de transferir esos conocimientos o estrategias a otras situaciones similares:

\footnotetext{
Metacognición significa el conocimiento de uno mismo concerniente a los propios procesos y productos cognitivos o a todo lo relacionado con ellos, por ejemplo, las propiedades de infomación o datos relevantes para el aprendizaje. Así, practico la metacognición (metamemoria, metaaprendizaje, metaatención, metalenguaje, etc.) cuando caigo en la cuenta de que tengo más dificultad en aprender A que B; cuando comprendo que debo verificar por segunda vez $\mathrm{C}$ antes de aceptarlo como un hecho; cuando se me ocurre que haría bien en examinar todas y cada una de las alternativas en una elección múltiple... [...] La metacognición indica, entre otras cosas, el examen activo y consiguiente regulación y organización de estos procesos en relación con los objetos cognitivos sobre los que versan, por lo general al servicio de algún fin u objetivo concreto. (Jiménez-Rodríguez, 2004, pp. 50-51)
}

De esta manera, la metacognición le permite al individuo poder tomar las mejores decisiones a la hora de resolver problemas por medio de la autorregulación. La conciencia metacognitiva permite determinar cuáles son las acciones apropiadas para lograr una determinada meta (Alhaqbani \& Riazi, 2012). Además, la metacognición implica identificar y comprender las distintas formas de influencia social que actúan sobre un individuo (JiménezRodríguez, 2004). 
Algunas afirmaciones propuestas por Flavell (citado en Jiménez-Rodríguez, 2004, p. 57) sobre el conocimiento metacognitivo son:

\footnotetext{
a. No hay ninguna razón para pensar que el conocimiento metacognitivo es diferente cualitativamente de cualquier otro tipo de conocimiento.

b. Algún conocimiento metacognitivo es declarativo y otro es procedimental.

c. Como cualquier proceso de adquisición de conocimiento, el conocimiento metacognitivo crece de forma lenta y gradual, a través de los años de experiencia en el dominio de la actividad cognitiva.

d. Puede ser activado automáticamente, como cualquier otro conocimiento.

e. Las bases del conocimiento metacognitivo pueden estar defectuosas como las bases de cualquier otro tipo de conocimiento.
}

Siguiendo estas afirmaciones, Pintrich (2002, p. 224) asegura que:

\footnotetext{
a. Los estudiantes tienen que conocer las estrategias para poder aplicarlas. Es decir, por sí mismos no se logra que ellos las apliquen.

b. Hay una transferencia de aprendizaje. Es la habilidad de utilizar el conocimiento aprendido en una situación, en otra totalmente distinta.
}

Sin embargo, como bien menciona Jiménez-Rodríguez (2004) hay que tener en cuenta una diferencia fundamental: una cosa es tener determinada información y ser capaz de acceder a esta y otra, muy distinta, es ser capaz de poseer una habilidad y lograr aplicarla exitosamente. La diferencia radica entre conocer los métodos y saber cuándo, dónde, cómo y por qué utilizarlos.

Ahora bien, aplicadas a la lectura, las estrategias metacognitivas son "el conocimiento de los lectores" (Alhaqbani \& Riazi, 2012, p. 233); es decir, la metacognición es usada por los lectores durante los procesos de lectura así como en los mecanismos de autocontrol usados para monitorear y fortalecer la comprensión.

En general, a nivel educativo, la metacognición permite que los estudiantes regulen su propio proceso de aprendizaje al monitorear y controlar sus pensamientos (Wade \& Reynolds, 1989; El-Hindi, 1997; Hall, 1991; Muhammand Al-Khateeb, 2011). El que los estudiantes estén conscientes de las actividades metacognitivas les ayudará a saber cuáles estrategias usar, cómo usarlas así como determinar por qué, cuándo y dónde usar una estrategia o la otra. En otras palabras, usarán conocimiento declarativo, procedimental y condicional (Alhaqbani \& Riazi, 2012). En resumen, el usar estrategias metacognitivas mejora el proceso de aprendizaje (Muhammand Al-Khateeb, 2011; El-Hindi, 1997).

\subsection{Componentes de la metacognición}

En esta sección se hablará sobre sobre los elementos que componen la metacognición: el conocimiento metacognitivo, el cual abarca el conocimiento relacionado con la persona, las estrategias empleadas y las tareas. Además, se expondrá sobre las experiencias, el monitoreo y el control metacognitivo.

El primer componente de la metacognición es el conocimiento metacognitivo, el cual a su vez, se desglosa en el conocimiento relacionado con la persona, las estrategias y las tareas a las cuales se enfrenta la persona.

\subsubsection{Conocimiento metacognitivo}

Es conocimiento declarativo basado en hechos o creencias sobre cómo ciertos factores o variables afectan el resultado de las tareas cognitivas. Incluye conocimiento 
general sobre cognición así como la consciencia y el conocimiento sobre la cognición propia (Pintrich, 2002). Se divide en:

a. Conocimiento relacionado con la persona: es el conocimiento sobre las habilidades, tanto las debilidades como las fortalezas (Pintrich, 2002). Se incluyen la motivación, el interés y el valor que tiene determinada tarea para el estudiante.

Sin embargo, puede haber creencias falsas que distorsionen o impidan el uso de estrategias metacognitivas: "Although self-knowledge itself can be an important aspect of metacognitive knowledge, it is important to underscore the idea that accuracy of selfknowledge seems to be most crucial for learning"1 (Pintrich, 2002, p. 222).

Trabajar en el autoestima de los estudiantes no es la solución ante las creencias falsas. En primer lugar, el autoestima es un constructo totalmente diferente del autoconocimiento de acuerdo con Pintrich (2002). En segundo lugar, se podría cometer el error de dar retroalimentación positiva pero falsa sobre las fortalezas y debilidades. Entonces, si los estudiantes no cuentan con una imagen clara sobre sus verdaderas debilidades, es poco probable que hagan un esfuerzo para adquirir o construir nuevo conocimiento: "Students who lack knowledge of their own strengths and weaknesses will be less likely to adapt to different situations and regulate their own learning in them"2 (Pintrich, 2002, p. 223).

b. Conocimiento relacionado con las estrategias: es el conocimiento general sobre estrategias para el aprendizaje, el pensamiento y la resolución de problemas (Pintrich, 2002). Implica que la persona esté consciente de que cada tarea requiere una habilidad mental especial (Muhammand Al-Khateeb, 2011); sin dejar de lado el poder seleccionar la estrategia más efectiva para lograr determinada meta según la tarea.

Por otra parte, hay que tomar en cuenta las condiciones que deben existir para usar una estrategia u otra (Flavell, 1979; Pintrich, 2002). Además, estas estrategias son generales, de manera que pueden ser aplicadas en la mayoría de las disciplinas académicas (Pintrich, 2002).

Las estrategias están relacionadas en cómo los estudiantes memorizan la información, extraen el contenido del texto y entienden lo que escuchan en una clase o leen un texto. También se incluye la habilidad de establecer submetas y monitorear la propia cognición: hacerse preguntas mientras van leyendo un texto, releer algo que no se ha entendido bien. Estas estrategias se dividen, según Weinstein \& Mayer, citados por Pintrich (2002, p. 224), en:

\footnotetext{
-Ensayo: repetir una y otra vez las palabras o términos para uno mismo. No es necesariamente la estrategia más eficiente para aprender procesos cognitivos más complejos.

-Elaboración: se incluyen estrategias mnemotécnicas como resumir, parafrasear y seleccionar las ideas principales de los textos. Estas estrategias de elaboración permiten un procesamiento más profundo del material, lo cual redunda en una mejor comprensión y aprendizaje en comparación con el obtenido por las estrategias de ensayo.

-Organización: se incluyen diferentes formas de resumir, organizar conceptos en un mapa y tomar nota. Permite que el estudiante logre vincular diferentes elementos del contenido entre sí.
}

c. Conocimientos relacionados con las tareas: los individuos acumulan conocimientos sobre las diferencias en las tareas, es decir, algunas tareas son más difíciles y pueden requerir diferentes estrategias. Reconocerlas les permite reconocer el "qué" y el "cómo", pero sobretodo deben saber "por qué" y "cuándo" usarlas: "An important aspect of learning about strategies is the knowledge of when and why to use them appropriately... Because not all strategies are appropriate for all situations, the learner must develop some knowledge of the different conditions and tasks where the different strategies are used most appropriately"3 (Pintrich, 2002, p. 221). 
Un punto importante a tomar en cuenta es el hecho de que la situación local o las normas sociales y culturales pueden condicionar el uso de determinadas estrategias: ya sean promovidas por un profesor que las esté enseñando o que haya una manera culturalmente más aceptable para resolver un problema.

\subsubsection{Experiencias metacognitivas}

Se refieren a experiencias cognitivas conscientes o afectivas relacionadas con cualquier labor intelectual. Son las reflexiones realizadas mientras se ejecuta una tarea cognitiva (Jiménez-Rodríguez, 2004).

\subsubsection{Monitoreo metacognitivo}

En este se evalúa el estado de un proceso de alguna actividad metacognitiva (Pintrich, 2002).

\subsubsection{Control metacognitivo}

Permite regular una actividad cognitiva en proceso, ya sea: deternerse, continuar o cambiar lo que se ha estado haciendo (Jiménez-Rodríguez, 2004).

\subsection{Procesos metacognitivos}

El emplear adecuadamente las estrategias metacognitivas implica seguir un proceso. Ahora bien, este proceso se divide en la planificación, la supervisión y una evaluación final. El hecho de que cada parte esté separada de la otra no implica que se pueda prescindir de una etapa, o que no se pueda devolver a un estadio anterior.

\footnotetext{
a. La planificación del aprendizaje: conocer la naturaleza de la tarea; saber qué se domina y qué no con el fin de poder relacionar la nueva información con otra; establecer objetivos a corto plazo así como decidir cuáles estrategias va a seguir para lograr sus metas

b. La supervisión sobre el proceso de aprendizaje, también llamado monitoreo: el estudiante es capaz de regular su propio proceso

c. La evaluación del éxito o no del aprendizaje y de la aplicación de las diferentes estrategias, es decir, es ser capaz de determinar cuán exitoso fue todo el proceso de aprendizaje, tomando en cuenta las dificultades encontradas (Jiménez-Rodríguez, 2004, p. 57)
}

Ahora bien, Jiménez-Rodríguez (2004) plantea que dichas funciones se pueden agrupar en tres dimensiones: la planificación, el monitoreo y la evaluación. Muhammand Al-Khateeb (2011) añade una más: el control.

En el primer proceso, la planificación, se determina el objetivo relacionado con el problema, la naturaleza del problema y la estrategia más apropiada. Es necesario tomar en cuenta los conocimientos previos y cuáles de esos conocimientos ayudarán a resolver la tarea (Jiménez-Rodríguez, 2004). Algunas de las habilidades ubicables en esta etapa según Jiménez-Rodríguez (2004, p. 57) son:

\footnotetext{
a. determinar con precisión el objetivo,

b. determinar los conocimientos previos que serán de utilidad,

c. escoger la estrategia ejecutiva que se debe seguir,

d. organizar los pasos en un orden serial,

e. determinar obstáculos probables y potenciales errores,
} 
f. determinar métodos para tratar las dificultades y los errores,

g. determinar el tiempo para resolver la tarea,

h. predecir los resultados deseados o esperados.

Como indica Muhammand Al-Khateeb (2011), los estudiantes pueden aplicar estas habilidades si al realizar una tarea se preguntan: ¿cuál es el objetivo de esta tarea?, ¿cuáles procedimientos o estrategias puedo utilizar para lograr la tarea?, ¿cuáles son los problemas y errores que pueda encontrar?, ¿cuánto tiempo me va a tomar lograr esta tarea?

Para El-Hindi (1997), las estrategias metacognitivas en una etapa de planeamiento son: identificar el propósito de la lectura, activar conocimiento previo, ver un anticipo del texto y hacer predicciones del texto.

En el segundo proceso, el de monitoreo y control se incluyen las siguientes habilidades citadas por El-Hindi (1997, p. 10):

\footnotetext{
a. concentrarse en los detalles del objetivo o saber qué se está haciendo,

b. comprometerse con los procedimientos y los pasos que deben seguirse serialmente,

c. darse cuenta cuándo se alcanzan los subobjetivos,

d. saber cuándo moverse al siguiente paso,

e. saber qué información es importante y que debe ser recordada,

f. escoger los procedimientos apropiados que están relacionados con el contexto,

g. explorar las dificultades y los errores,

h. saber cómo superar las dificultades y saber lidiar con los errores.
}

Los estudiantes pueden aplicar estas habilidades de monitoreo y control al preguntarse a sí mismos para constatar los pasos anteriores, durante la ejecución de una tarea. No en vano, El-Hindi (1997) llama a esta segunda etapa "etapa borrador".

Según El-Hindi (1997, p. 10) el tercer proceso, la evaluación, incluye:
a. evaluar hasta qué punto se ha logrado el objetivo,
b. juzgar la eficiencia y la precisión de los resultados,
c. evaluar cuán apropiados fueron los métodos empleados,
d. evaluar cómo se trataron los obstáculos y los errores,
e. evaluar la efectividad del plan y su implementación

Los estudiantes pueden aplicar estas habilidades al preguntarse: ¿se lograron los objetivos de la tarea?, ¿el método que seguí fue exitoso?, ¿debería seguir el mismo método la próxima vez?, ¿cuál fue el método más exitoso?, ¿los problemas predichos existieron?, ¿cómo fueron superados?

\subsection{Variables metacognitivas}

La metacognición no son solo los procesos mencionados anteriormente. Las publicaciones realizadas por Jiménez-Rodríguez (2004) y Rossi (1991) demuestran que hay cuatro variables que deben ser tomadas en cuenta al usar o enseñar las estrategias metacognitivas.

\subsubsection{La persona}

Esta variable tiene que ver con las características de la persona: los conocimientos previos, los intereses, las limitaciones, la edad, las habilidades, la motivación, el conocimiento del proceso de la tarea, el uso y el control de las estrategias (Jiménez-Rodríguez, 2004). 


\title{
1.3.2 La tarea
}

Es el conocimiento sobre cómo la naturaleza y las demandas de la tarea influyen en la ejecución. En este aspecto se incluye: la ambigüedad, el propósito planeado, las estrategias necesarias, la atención y el esfuerzo (Jiménez-Rodríguez, 2004). La tarea es una de las variables más importante pues estructura la actividad del sujeto, define el contenido de las representación y por ende las operaciones que deben ser llevadas a cabo. Por otro lado, las tareas deben de ser precisas y deben, en cierta manera controlar lo que el sujeto puede hacer, al menos, parcialmente (Rossi, 1991).

Muchas veces, se considera que las tareas tiene una relación directa con evaluación, es decir, que en ciertas tareas de comprensión de lectura se logra evaluar una determinada habilidad de lectura como resumir, contestar preguntas de comprensión o resumir. Sin embargo, muchas veces las operaciones implementadas por el sujeto no necesariamente son las que se utilizarían en una tarea de comprensión de lectura:

\begin{abstract}
In defining a task model, the nature of the task presented in instructions to the subject must be distinguished from the type of test used to measure performance. The three main tasks generally assigned to subjects are comprehension, retention and summarizing. These tasks are combined with testing, which is normally in the form of comprehension questions, measures of recall, and summarizing. Although this list suggests there is an one-to-one relationship between task and test, there are numerous examples in the literature where a recall test is associated with a comprehension task or the contrary, where comprehension is measure through recall, despite the fact that the operations implemented by subject during retention of a text clearly differ from those employed in text comprehension. [...] the task assigned to the subject through explicit instructions may differ from the task the subject assigns to him/herself. Instructions and design should be planned in such ways as to make the two coincide as closely as possible. (Rossi, 1991, pp. 5-6)
\end{abstract}

\subsubsection{El contexto}

Jiménez-Rodríguez (2004) cita a Mayor, Suengas y González (1995) quienes hacen una distinción entre el contexto potencial (todas las posibilidades de acción) y el relevante (el que ubica la actividad, construido por el individuo). El contexto facilita o interfiere a la hora de establecer la congruencia de la actividad metacognitiva, limita la interacción del sujeto con el ambiente, selecciona o extrapola los significados relevantes para la actividad metacognitiva (Jiménez-Rodríguez, 2004).

\subsubsection{Las estrategias}

Las estrategias son procesos secuenciales usados por el individuo para controlar una actividad metacognitiva con el fin de lograr una meta. Como regla general, el tipo de estrategia usada por el lector depende de la estructura del texto, del conocimiento del lector así como de la naturaleza de la tarea propuesta (Rossi, 1991). Ahora bien, una misma estrategia puede ser beneficiosa para una persona, al permitirle lograr un buen desempeño, pero eso no necesariamente será el caso para otras personas, pues hay otras variables que deben ser tomadas en cuenta.

En cuanto al papel de la metacognición en las estrategias, no solo es darse cuenta de lo que se sabe o no, es conocer qué se puede hacer para solucionar los problemas o los fallos de comprensión.

Algunas estrategias para desarrollar comportamientos metacognitivos son, según Jiménez-Rodríguez (2004, p. 40): 
a. Identificar lo que sabe y lo que no.

b. Hablar sobre el pensamiento: durante procesos de instrucción el docente puede nombrar los procesos de pensamiento.

c. Autoevalución.

Esta misma autora menciona que Baker y Brown, en 1984, señalaron cuatro estrategias metacognitivas básicas:

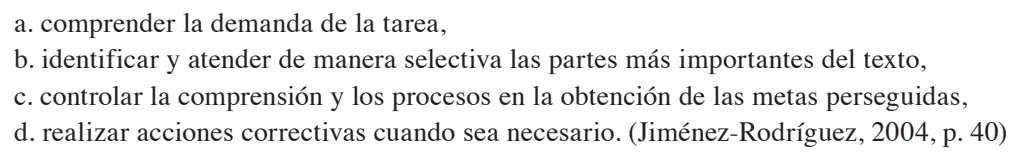

\section{Aplicar las estrategias metacognitivas en la clase de español L2 o ELE}

Tomando en cuenta los dos principales temas del apartado introductorio, desde la perspectiva metacognitiva, un buen lector es quien: "modifica el proceso de lectura además de usar estrategias según las demandas textuales" (Alhaqbani \& Riazi, 2012, p. 233).

Enseñar a los estudiantes a utilizar las estrategias metacognitivas es la base para el desarrollo de la conciencia metacognitiva. Esta conciencia es la base del aprender a aprender y a comprender, es enseñarle pensamiento estratégico a los alumnos (Jiménez-Rodríguez, 2004).

Ahora bien, ¿cómo pueden enseñarse estas estrategias en una clase de segunda lengua o lengua extranjera, en este caso, el español?, ¿es realmente útil hacerlo?, ¿se podría asumir que existe una transferencia total de habilidades de la primera lengua del estudiante hacia la segunda o hacia la lengua que está aprendiendo?

En primer lugar, esa transferencia de habilidades se da cuando se ha logrado cierto nivel de habilidad lingüística, según la hipótesis de la interdependencia lingüística (Yamashita, 2004). Carrell, Carson \& Zhe (1993) lograron demostrar que hablantes nativos y no nativos lograban un desempeño similar en lenguas como el chino y el inglés al usar estrategias similares. Sin embargo, esa transferencia se daba siempre y cuando el estudiante las hubiera aprendido en su lengua materna: situación que no necesariamente describe a la mayoría de aprendices de lenguas extranjeras.

A pesar de que el proceso pueda tomar tiempo, la enseñanza de estrategias metacognitivas en otra lengua permite desarrollar la autonomía del estudiante. El aprendiz será capaz de establecer sus propios objetivos, saber qué estrategia puede aplicar y cómo, evaluar su propio proceso de aprendizaje, evaluar los resultados obtenidos — sean estos los esperados o no- así como determinar qué tanto se logró con respecto a las expectativas iniciales. Por otro lado, promueve el desarrollo de actitudes positivas y permite el desarrollo de una conciencia sobre la propia eficiencia. El Instituto Cervantes (2008) asegura que: "El conocimiento sobre la metacognición ha sido de gran valor en la enseñanza de lenguas extranjeras, hasta el punto en que los procesos metacognitivos han pasado a formar parte de los contenidos de los programas de enseñanza junto con el resto de conocimientos (lingüísticos, pragmáticos y culturales)" (p. 356).

A continuación se plantea una serie de recomendaciones para aplicar las estrategias metacognitivas a la hora de resolver tareas de comprensión de lectura, tomando en cuenta los procesos y las variables expuestas anteriormente. Estas estrategias se planean para un público adulto que aprende español como lengua extranjera. Puede ser implementadas en clases sin 
importar el nivel de adquisición de lengua alcanzado, pues al ser proceso se puede ir haciendo gradualmente. No obstante, estudiantes que están en un nivel B1 en adelante, según el Marco Común Europeo de Referencia para las Lenguas son quienes podrán tener un mayor provecho de estas estrategias pues lo textos son más extensos. Lo ideal sería trabajar con comprensión de lectura una o dos veces por semana, pero eso dependerá de la división curricular de las clases, el programa o las necesidades del estudiante. Ahora bien, a la hora de abordar cualquier texto, se debe tomar en cuenta estos pasos expuestos a continuación. En general, al principio el proceso es más guiado pues muchos estudiantes no están acostumbrados a utilizar las estrategias metacognitivas, pero con tiempo y práctica ellos deberían empezar a automatizar los pasos expuestos a continuación:

\subsection{Planificación}

En la planificación es necesario saber cuál es el objetivo, determinar para qué se lee ese texto y cuál es el fin último; puede ser contestar una serie de preguntas que responden a una lectura general, específica o inferencial, dependiendo de las existencias y planteamiento de la tarea. Es importante no dar el material de lectura a los estudiantes y dejarlos solo, debe haber técnicas previas para motivar al estudiante así como para activar todo el conocimiento previo que tengan.

Es recomendable la activación de conocimientos previos: en un salón de clase se puede recurrir a una lluvia de ideas sobre una frase o una lista de vocabulario revisada anteriormente. También se pueden emplear a estrategias de trabajo cooperativo para resolver una serie de preguntas por medio del uso de la tecnología en la clase para extraer información, incluso hacer una pequeña investigación previa.

Es recomendable que al exponer a los estudiantes a un tipo de tarea y de lectura específico, se les guíe sobre cuáles estrategias son las más apropiadas y cuáles no, explicando, claro está, la razón: ante preguntas inferenciales no se puede hacer una lectura superficial, pero sí se puede hacer un primer acercamiento escaneando el texto para encontrar palabras clave, sinónimos o parafraseo a la pregunta. Se recomienda explicarles que cuando realizan un examen, el tiempo es un factor importante, de manera que leer las preguntas antes de abordar el texto es una manera más eficiente de solucionar las tareas propuestas. En algunos casos, sobre todo en tareas de clozed, leer el texto incompleto es una buena idea antes de completarlo con las palabras o frases faltantes. Además, es adecuado en esta primera etapa de mecanización de estrategias empezar a guiarlos en el monitoreo metacognitivo que se explica en el apartado siguiente.

\subsection{Monitoreo y control}

Es necesario que los estudiantes se enfoquen en el objetivo de la tarea por cumplir. Si se ha determinado cuál es el objetivo de la tarea en el paso anterior — planificación - se deben haber establecido las estrategias para resolver esa tarea: en este punto la clave está en seguir esos pasos serialmente.

Ahora bien, lograr el objetivo final es posible por medio del cumplimiento de submetas. Es decir, cada paso serial debe llevar al estudiante al logro de pequeños objetivos. Si uno de esos subobjetivos no se cumple esto le permite al estudiante, quien está monitoreando activamente sus procesos cognitivos, detenerse y devolverse: puede que la estrategia escogida 
no sea la mejor, puede que necesite más conocimientos previos, puede que su esquema no coincida con el propuesto por el autor.

También es en esta fase cuando el aprendiz debe detectar el momento de moverse a una siguiente fase o cambiar la estrategia de comprensión de lectura usada en ese momento, por ejemplo, pasar de un escaneo a una lectura con mayor profundidad.

Además, también al monitorear se debe ser capaz de detectar qué información es importante y cuál no, cuál información debe ser recordada, comparada con información ya existente y cuál, por el contrario, no debe ser tomada en cuenta: esto en lectura académica evaluativa es importante y la única guía para su correcto desenvolvimiento es el tipo de tarea propuesta.

Por otro lado, es importante saber lidiar con las dificultades, sean estas esperadas o no, y saber cómo sortearlas sin mayores contratiempos.

En resumen, esta fase de monitoreo y control es fundamental, pues nada se logra con una buena planificación si no se lleva a cabo y más aún si no se logra. Con el tiempo, la guía del docente y la práctica estos pasos serán mecanizados, pero para poder lograrlo, es necesario que se realicen los pasos uno por uno en el salón de clase.

\subsection{Evaluación}

Finalmente, en este tercer apartado, es necesario reconsiderar hasta qué punto se ha logrado el objetivo propuesto en la planificación, el cual ha sido establecido por medio de la tarea de comprensión de lectura. La eficiencia y la precisión de los resultados deben ser tomadas en cuenta. En un salón de clase, el docente podría facilitar una puesta en común sobre cómo los estudiantes sortearon dificultades y así comparar estrategias empleadas según las diferentes tareas y los diferentes textos a los cuales los estudiantes de lengua son expuestos, dependiendo del nivel de adquisición en el que se encuentren. La evaluación retoma en gran medida parte de los procesos mentales - consciencia metacognitiva- y se devuelve hasta el momento de la planificación: cuáles estrategias, cómo y por qué: estrategias metacognitivas.

Es importante no dejar de lado que el docente les explique a los estudiantes qué hacer en caso de no saber alguna palabra - enfocarse en el contenido global y tratar de apoyarse en el contexto, evitar la frustración de entrada-; reforzar que la idea de releer no es negativa y que en muchos casos favorece la comprensión; que es necesario ir haciendo predicciones de lo que podría aparecer en el texto y que leer superficialmente, no es una forma negativa de leer, si se toma en cuenta cuándo se debe leer de esa manera y cuándo es necesario profundizar en los detalles: lectura inferencial. Tener en cuenta lo anterior (las estrategias, los elementos que componen el conocimiento metacognitivo así como sus variables) y saber aplicarlo, tomando en cuenta los procesos mentales a la hora de leer y de realizar tareas de comprensión de lectura dentro y fuera del aula es lo que permitirá que los estudiantes desarrollen una conciencia metacognitiva: el pensar sobre esas estrategias a nivel de su propio pensamiento son las estrategias metacognitivas.

\section{Conclusión}

La implementación de las estrategias metacognitivas en un salón de clase es una tarea que podría implicar un arduo trabajo por parte del docente pues implica un proceso de preparación más largo: es necesario seleccionar bien los textos, escoger las tareas y saber 
presentarlo en la clase. Si se toma en cuenta que esta población estudiantil además de lidiar con una propuesta que les puede ayudar a mejorar su rendimiento, está aprendiendo una segunda lengua, puede que la tarea tome un matiz aún más complejo.

No obstante, las ventajas son muchas. El emplear estrategias metacognitivas permite el desarrollo de actitudes positivas hacia el aprendizaje de la lengua y hacia ellos mismos como aprendices de esa lengua, lo cual está directamente relacionado con el área afectiva de los estudiantes. Además, los estudiantes podrán tomar conciencia de su propia eficiencia y efectividad a la hora de resolver tareas de comprensión de lectura: sean estas meros ejercicios en clase o que tenga valor evaluativo importante para su desarrollo académico. Por otro lado, contar con estrategias y saber emplearlas en un marco metacognitivo les permite desarrollar la autonomía del estudiante, en el cual, es él o ella los protagonistas de su propio proceso de aprendizaje: ellos mismos están autogestionando sus propios procesos: el profesor es el facilitador de estrategias y de contextos o situaciones en las cuales se promueva el uso de la conciencia metacognitiva aplicada en forma de estrategias.

\section{Notas}

1. "A pesar de que el conocimiento sobre el sí mismo puede ser un aspecto importante del conocimiento metacognitivo, es importante subrayar la idea de que la precisión del conocimiento sobre el sí mismo parece ser el más crucial para el aprendizaje" (Pintrich, 2002, p. 222).

2. "Los estudiantes que carecen de conocimiento de sus propias fortalezas y debilidades serán menos propensos a adaptarse a situaciones diferentes y regular su propio aprendizaje en dichas situaciones" (Pintrich, 2002, p. 223).

3. "Un aspecto importante sobre el aprender sobre estrategias es saber cuándo y por qué usarlas apropiadamente... Dado que no todas las estrategias son apropiadas para todas las situaciones, el aprendiente debe desarrollar algún conocimiento de las diferentes condiciones y tareas en las cuales las diferentes estrategias son usadas más apropiadamente" (Pintrich, 2002, p. 221).

\section{Bibliografía}

Alhaqbani, A. \& Riazi, M. (2012). Metacognitive awareness of reading strategies use in Arabic as a second language. Reading as a Foreign Language. 24 (2), 231-255.

Antonijevic, N. y Chadwick, C. (1981/82). Estrategias cognitivas y metacognición. Revista de Tecnología Educativa. 7, 4, 307-321.

Brantmeier, C. (2003). Does gender make a difference? Passage content and comprehension in second language reading. Reading in a Foreign Language. 15 (1), 1-27.

Carrell, P., Carson, J.G. \& Zhe, D. (1993). First and Second Language Strategies: Evidence from Cloze. Reading in a Foreign Language. 10 (1), 953-965.

Cuetos-Vega, F. (2000). Psicología de la lectura: diagnóstico y tratamiento de los transtornos de lectura. Barcelona: CISSPRAXIS.

Dunlosky, J. y Metcalfe, J. (2009). Metacognition. Estados Unidos de América: Sage Publications, Inc.

El-Hindi, A. (1997). Connecting Reading and Writing: College Learners Meta Cognitive Awareness. Journal of Developmental Education. 21 (2), 10-17. 
Farrell, T. S. C. (2001). Teaching Reading Strategies: 'It Takes Time!'. Reading in a Foreign Language. 13 (2), 631-646.

Flavell, J. H. (1979). Metacognition and Cognitive Memory: a New Area of CognitiveDevelopmental Inquiry. American Psycologist. 34 (10), 906-911.

Graesser, A. C., Olde, B., Pomeroy, V., Whitten, S., Lu, S. \& Craig, S. (2006). Inferencias y preguntas en la comprensión de textos científicos. Tarbiya: Revista de Investigación e Innovación Educativa del Instituto Universitario de Ciencias de la Educación. Universidad Autónoma de Madrid. 36, 103-128.

Hall, W. S. (1991). La comprensión de la lectura. Por A. Puente (Ed.). Compresión de la lectura y acción docente. (25-72). España: Ediciones Pirámide.

Jiménez-Rodríguez, V. (2004). Metacognición y comprensión de la lectura: evaluación de los componentes estratégicos (procesos y variables) mediante la elaboración de una escala de conciencia lectora (ESCOLA) (Tesis doctoral). Universidad Complutense de Madrid.

Muhammand Al-Khateeb, O. S. (2011). The Impact of Using Meta Cognitive Learning Strategies on Al-Hussain Bin Talal University Students' Achievement in and Attitudes towards Health Concepts in "Tenet of Worship" Course. Asian Social Science. 7 (3), 165-186.

Navarro, A. (1991). Procesos perceptivos y atencionales durante la lectura. Por A. Puente (Ed.). Compresión de la lectura y acción docente. (41-72). España: Ediciones Pirámide.

Pintrich, P. R. (2002). The Role of Metacognitive Knowledge in Learning, Teaching, and Assessing. Theory Into Practice. 41 (4), 219-225.

Puente, A. (1991). Comprensión de lectura y acción docente. Madrid: Pirámide.

Rossi, J-P. (1991). Input and Output: Processing and representation. Por G. Denhière \& J-P. Rossi. (Eds.). Advances in Psychology 79: Text and Text Processing. (3-18). Holanda: Elsevier Science Publishers B.V.

Rumelhart, D. E. (2013). Toward an Interactive Model of Reading. Por D. E. Alvermann, N. J. Unrau \& R. B. Ruddell (Eds.). Theoretical models and processes of reading. (719-747). Newark, DE: International Reading Association.

Sainz, J. S. (1991). Procesos de lectura y comprensión de lenguaje. Por J. Mayor \& J. L. Pinillos (Eds.). Tratado de Psicología General. (641-738). Madrid: Alhambra Longman http:// es.scribd.com/doc/5199431/PROCESOS-DE-LA-LECTURA.

Wade, S. E. \& Reynolds, R. E. (1989). Developing metacogntive awareness. Journal of Reading. 33 (1), 6-14.

Yamashita, J. (2004). Reading attitudes in L1 and L2, and their influence on L2 extensive reading. Reading in a Foreign Language. 16 (1), 1-19.

Yang, Y-F. (2002). Reassessing Readers' Comprehension Monitoring. Reading in a Foreign Language. 14 (1), 18-42. 
